Mittheilungen aus dem chemischen Laboratorium der Universität Marburg,

(Eingelaufen am 31. Juli 1905.)

Ueber die Einwirkung von Brom und von Chlor auf Phenole: Substitutionsproducte, Pseudobromide und Pseudochloride;

ron $T h$. Zincke.

[Vierzehnte Mitheilung.]

Ueber die Einwirkung von Brom auf p-Diphenoldimethylmethan: Pseudobromide und Chinone des p-Isopropylphenols; von Th. Zincke und M. Grïters $\left.{ }^{1}\right)$.

Nach Dianin spaltet sich das Diphenoldimethylmethan:<smiles>CCCCC(C)(C)C1CCCCC1</smiles>

beim Schmelzen mit Alkali in Phenol and Isopropylphenol, und in ähnlicher Weise soll sich die homologe Verbindung, das Diphenoldiäthylmethan, beim Erbitzen mit rauchender Salzsäure verhalten ${ }^{2}$ ).

Diese Angaben haben uns veranlasst, das Diphenoldimethylmethan in den Kreis der Untersuchungen zu ziehen, wir durften hoffen, dass auch durch Einwirkung von Brom eine derartige Spaltung eintreten und sich Pseudobromide des Isopropylphenols bilden würden.

1) Zum Theil ans der Dissertation Marburg 1901.

9) Ber. d. deutsch. chem. Ges. 25, Ref. 334 . 
Diese Erwartung hat sich bestätigt, wir haben zwei verschiedene Pseudobromide darstellen können, welche schöne und sehr beständige Methylenchinone lieferten.

Lässt man Brom in Eisessiglösung auf das durch Condensation von Phenol und Aceton leicht darstellhare Diphenoldimethylmethan einwirken, so findet zunächst Eintritt von vier Atomen Brom statt. Die entstehende Tetrabromverbindung zeigt das Verhalten eines normalen Phenolderivates, sie ist ohne Veränderung in Alkali löslich; ihre Constitution wird jedenfalls durch die Formel:

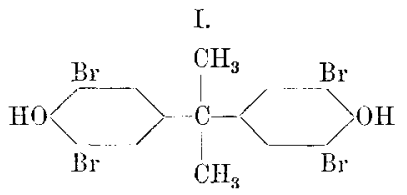

auszadrücken sein. Mit Natriumnitrit behandelt, tauscht die Verbindung zwei At. Brom gegen zwei Nitrogruppen aus und geht in ein Dibromdinitroderivat:

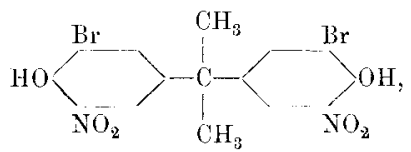

über.

Wird das Tetrabromid (I) obne Lösungsmittel der weiteren Einwirkung von Brom unterworfen, so tritt Spaltung des Moleküls ein, es bildet sich symmetrisches Tribromphenol und je nach den Bedingungen ein Pseudobromid mit sechs respective sieben At. Brom: $\mathrm{C}_{9} \mathrm{H}_{6} \mathrm{Br}_{6} \mathrm{O}$ oder $\mathrm{C}_{9} \mathrm{H}_{5} \mathrm{Br}_{7} \mathrm{O}$; in der Kälte entsteht vorzugsweise die erste, bei höherer Temperatur und überschüssigem Brom die zweite Verbindung.

Was den Verlauf der Reaction angeht, so ist es wohl das Wahrscheinlichste, dass sofort, entsprechend der Gleichung:

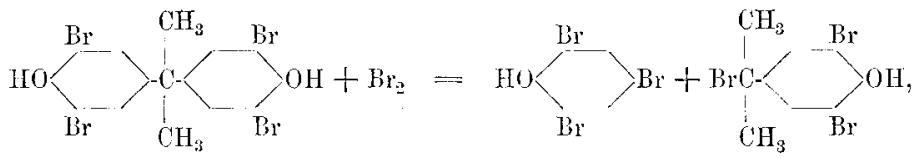


die Spaltung erfolgt und dann erst der weitere Eintritt von Brom in Haupt- und Seitenkette des Isopropylphenols stattfindet. Jedenfalls tritt vor der Spaltung keine weitere Kernsubstitution ein, sonst könnte nicht Tribromphenol entstehen, sondern es hätte sich ein höher gebromtes Phenol bilden müssen.

Es ist aber auch möglich, dass bei der weiteren Einwirkung des Broms zunächst die Seitenkette des Tetrabromids (I) angegriffen wird, der entstehende Bromwasserstoff die Spaltung herbeiführt und dann die weitere Kernsubstitution stattfindet. Wir haben diese Fragen nicht entscheiden können, da es nicht möglich war, Zwischenproducte zu fassen.

Bezüglich der Constitution der beiden Pseudobromide: $\mathrm{C}_{9} \mathrm{H}_{6} \mathrm{Br}_{6} \mathrm{O}$ and $\mathrm{C}_{9} \mathrm{H}_{5} \mathrm{Br}_{7} \mathrm{O}$ fragt es sich zunächst, wie viel Bromatome in den Kern eingetreten sind. Wir haben versucht, durch Oxydation der Bromide mit Salpetersäure diese Frage zu lösen, wir hofften zu gebromten Benzochinonen zu gelangen, haben aber kein derartiges Product beobachten können. Das Hexabromid oxydirt sich leicht unter Bildung harziger Producte, das Heptabromid wird viel schwieriger angegriffen, lässt sich aber in einen weissen, krystallinischen Körper überführen, dessen Natur bis jetzt nicht aufgeklärt worden ist. Auch verschiedene Umwandlungsproducte der Pseudobromide haben wir mit Salpetersäure behandelt, niemals aber ein Benzochinonderivat erhalten, auch nicht aus denen mit ungesättigter Seitenkette. Es treten hier augenscheinlich sehr verschiedenartige Reactionen ein, die einer besonderen Untersuchung werth sein dürften.

In dem Heptabromid sind jedenfalls alle Wasserstoffatome des Kernes durch Brom ersetzt worden, die Seitenkette enthält also noch drei Bromatome, von denen eins am tertiären Kohlenstoffatom stehen muss, da sonst die Verbindung kein Pseudobromid sein könnte; fraglich erscheint also nur noch die Stellung der beiden anderen Bromatome.

Wir haben diese Stellung nicht direct nachweisen können, aber aus dem Verhalten des Menthons bei der Bromirung darf der Schluss gezogen werden, dass das Heptabromid die Gruppe 
$\mathrm{CHBr}_{2}$ enthält. Wie v. Baeyer und Seuffert ${ }^{3}$ ) gezeigt haben, geht das Menthon beim erschöpfenden Bromiren in der Kälte in ein Hexabromid, $\mathrm{C}_{10} \mathrm{H}_{8} \mathrm{Br}_{6} \mathrm{O}$, über, welches ohne Frage unserem Heptabromid entspricht. Nach v. Baeyer und Seuffert kommt ihm - als Phenol aufgefasst -- die Formel II zu, woraus für die Heptabromverbindung die Phenolformel III folgen würde:

II.

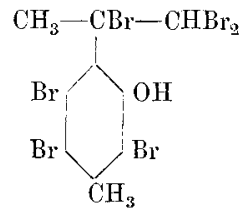

111 .

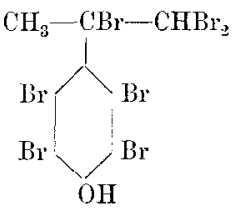

Auch das Hexabromid, $\mathrm{C}_{9} \mathrm{H}_{6} \mathrm{Br}_{6} \mathrm{O}$, dürfte vier At. Brom im Kern enthalten und sich nur dadurch vom Heptabromid unterscheiden, dass es an Stelle von $\mathrm{CHBr}_{2}$ die Gruppe $\mathrm{CH}_{2} \mathrm{Br}$ enthält (Formel IV):

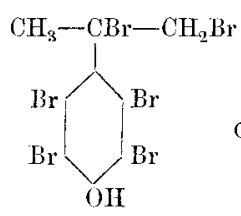

IV.

oder<smiles>C[Ge](Br)CBr</smiles><smiles>BrC=[In]CBr</smiles><smiles>Br[Mg]Br</smiles>

Für diese Annahme sprechen mancherlei Beobachtungen, ihre Richtigkeit hat aber noch nicht bewiesen werden können; es ist bis jetzt nicht gelungen das Hexabromid in irgend einer Weise direct mit dem Heptabromid zu verknüpfen. Hoffentlich bringt die weitere Untersuchung des Hexabromids diese Frage zur Entscheidung; bis dahin sind die hier gegebenen Formeln, soweit die Stellung der Bromatome in Betracht kommt, nur als vorläufige anzusehen. Im experimentellen Theile gehen wir auf das Hexabromid und seine Umwandlungsproducte jetzt noch nicht ein.

Beide Pseudobromide spalten leicht Bromwasserstoff ab und gehen in Methylenchinone über, welche als Penta- und Hexabromisopropylidenchinone (V und VI) bezeichnet werden können:

3) Ber. d. deutsch. chem. Ges. 34, 40 (1901). 
Sulstitutionsproducte, Pseudobromide und Pseudochloride. 79

V.<smiles>CC(CBr)=C(Br)Br</smiles>

VI.

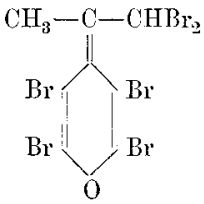

Diese Isopropylidenchinone sind sehr beständig, viel beständiger wie die aus Isoeugenol dargestellten Propylidenchinone ${ }^{4}$ ), sie lassen sich aus verschiedenen Lösungsmitteln umkrystallisiren und ohne Veränderung aufbewahren. Im merkwürdigen Gegensatze steht dazu die Unbeständigkeit des Chinons aus dem Hexabromid des Menthons (II), v. Baeyer und Seuffert haben es nicht isoliren können, sein Auftreten liess sich aber bei verschiedenen Reactionen durch die Rothfärbung erkennen. In der Menthonreihe handelt es sich allerdings um ein Orthochinon und es wäre möglich, dass diese reactionsfähiger und damit unbeständiger sind wie die p-Methylenchinone ${ }^{5}$ ).

Bei einigen Reactionen zeigen die beiden Isopropylidenchinone (V und VI) durchaus das Verhalten der Methylenchinone, bei anderen weichen sie davon ab, was wohl darauf zurückzuführen ist, dass auch in der Seitenkette leicht Aenderungen eintreten.

Mit Essigsäureanhydrid reagiren die Chinone in normaler Weise, also unter Anlagerung von einem Mol. Anhydrid, nur in der Kälte und bei Gegenwart von etwas Schwefelsäure. Es entstehen die Verbindungen:
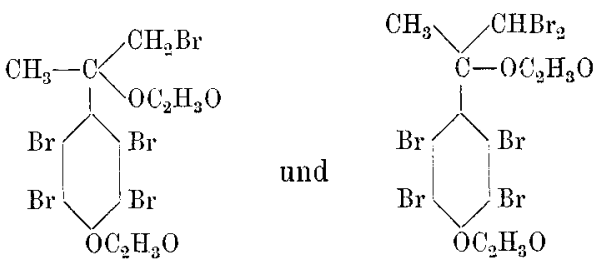

4) Diese Annalen $\mathbf{B 2 9}, 1$ (1903).

5) Nach neteren Versuchen von Fries und Hïbner scheint das nicht der Fall zu sein (mündliche Nittheilung). 
Lässt man aber das Anhydrid in der Hitze einwirken, so findet Abspaltung von Bromwasserstoff respective Brom statt und man erhält bromärmere Acetylderivate, welche auch aus den Pseudobromiden dargestellt werden können; sie sind unten näher erörtert.

Eine Addition von Essigsäure an die Chinone ist nicht gelungen; unter gewissen Bedingungen wirkt allerdings Essigsäure auf das Hexabromchinon (VI) ein, es entsteht aber nicht das Essigsäureproduct, sondern neben anderen Verbindungen das Wasserproduct (vergl. den experimentellen Theil).

Sehr eigenartig und kaum verständlich ist auch das Verhalten des Hexabromchinons gegen wasserhaltiges Aceton. Eine Wasseranlagerung, welche zu dem zugehörigen Phenolalkohol hätte führen müssen, findet nicht statt, sondern nur ein Austausch von Brom gegen Wasserstoff. Das Chinon $\mathrm{C}_{9} \mathrm{H}_{4} \mathrm{Br}_{6} \mathrm{O}$ geht in ein ungesältigtes Phenol, $\mathrm{C}_{9} \mathrm{H}_{5} \mathrm{Br}_{5} \mathrm{O}$, über. Die Reaction verläuft aber nicht sehr glatt.

Viel leichter lässt sich das Phenol $\mathrm{C}_{9} \mathrm{H}_{5} \mathrm{Br}_{5} \mathrm{O}$, welchem seinem Verhalten nach die Formel:

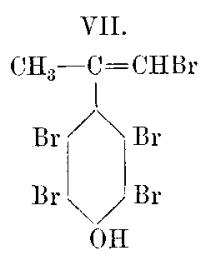

zukommen muss, mit Hülfe von Ziunchlorür aus dem Hexabromchinon (VI) darstellen. Hier handelt es sich um einen Vorgang, wie er auch bei Derivaten des $p$-Aethylphenols beobachtet worden ist, welche durch Reduction in Derivate des $p$-Vinylphenols übergehen ${ }^{6}$ ); man muss zunächst Addition von zwei At. Wasserstoff und dann Abspaltung von Bromwasserstoff annehmen :

$\left.{ }^{6}\right)$ Zincke, diese Annalen 322, 182 (1902). 
Snlstitutionsproducte, Pseudobromide und Pseudochloride. 81

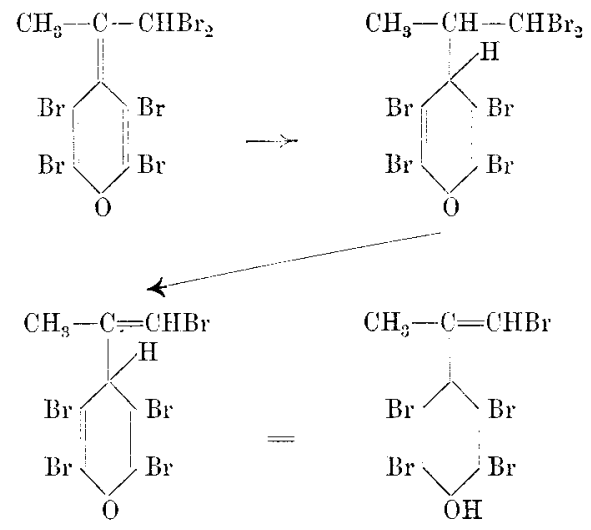

Die Einwirkung von Aceton wird man aber schwerlich in gleicher Weise deuten können; auch ein directer Austausch von Brom gegen Wasserstoff und darauffolgende Umlagerung erscheint wenig wahrscheinlich.

Als ungesättigte Verbindung addirt das $\mathrm{Phenol}_{9} \mathrm{H}_{5} \mathrm{Br}_{5} \mathrm{O}$ (VII) sowohl Brom als auch Bromwasserstoff. Im ersten Falle entsteht das Heptabrompseudobromid (III), im zweiten Falle erwarteten wir die Bildung des Hexabrompseudobromids (IV) und damit eine Verknüpfung des Hexabromids mit dem Heptabromid. Wahrscheinlich entsteht auch das Hexabromid, wir haben es aber nicht in reinem Zustande abscheiden können, sondera nur das Vorhandensein eines Pseudobromids durch Chinonbildung constatirt. Das Hauptproduct der Einwirkung von Bromwasserstoff auf das Phenol (VII) ist ein alkalilöslicher, gut krystallisirender Körper, dem vielleicht die Formel:

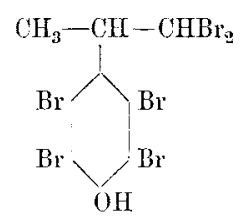

zukommt; untersucht ist er noch nicht. 
In ganz anderer Weise verläuft die Einwirkung von Aceton auf das Hexabromchinon (VI), wenn beim Kochen gefälltes Quecksilberoxyd zugesetzt wird, ein Versucb, der zu dem Zwecke angestellt wurde, um Abspaltung von Bromwasserstoff herbeizuführen. I)ie Entfärbung der Lösung tritt rasch ein; es wird aber kein Bromwasserstoff abgespalten, sondern das Chinon geht in das isomere ungesättigte Phenol über, dem die folgende Formel:

VIII.<smiles>C=C(C)C(Br)C(Br)C(Br)Br</smiles>

zukommt.

An Stelle von Aceton kann für diesen Versuch Alkohol angewendet werden, das Quecksilberoxyd ist es also, welches die Umwandlung bewirkt; in welcher Weise müssen wir dahingestellt sein lassen ${ }^{7}$ ).

Leichter lässt sich die gleiche Umwandlung durch Alkali in Aceton- oder Alkohollösung erreichen, am besten durch eine alkalische Zinnlösung; irgend eine Reduction findet lierbei nicht statt. Möglicherweise handelt es sich bei diesen Reactionen nur um eine einfache Umlagerung des Chinous in das Phenol ${ }^{8}$ ), wahrscheinlicher erscheint es uns aber, dass zunächst Anlagerung ron Wasser und Bildung des chinoiden Wasserproductes stattfindet und dann wieder Wasser austritt:

7) Beim Kochen mit Bleioxyd tritt die Umwandlung nicht eiu, ebensowenig bei Gegenwart von Schwefelsäure.

s) v. Baeyer und Seuffert nehmen in der Menthonreilie eine solche Umlagerung an; das Menthonhexabromid (Formel II) geht beim Schütteln sciner ätherischen Lösung mit Soda unter Abspaltung von Bromwasserstoff in ein ungesättigtes Phenol über. Als Zwischenproduct entstoht das Chinon und dieses soll sich $11 \mathrm{mlagern.}$ 


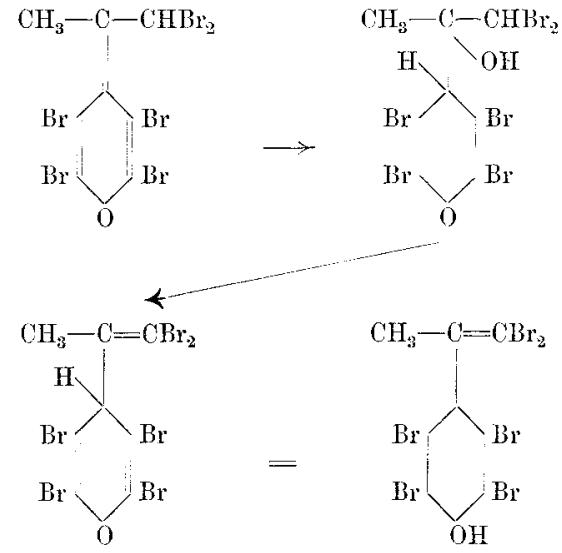

Für diese Erklärung spricht das Verhalten des aus dem Chinon erhaltenen Diacetats (a) bei der Verseifung ${ }^{9}$ ); man erhält nicht die entsprechende Oxyverbindung (b), sondern die ungesättigte Verbindung (VIII); die Seitenkette verliert Wasser:

a.<smiles>CCOC(C)(CC)C(Br)C(Br)Br</smiles>

b.<smiles>CC(Br)(Br)C(Br)Br</smiles><smiles>CC(C)(C(Br)Br)C(Br)C(Br)Br</smiles>

Sehr leicht erhält man das ungesättigte Phenol auch durch Verseifen des Heptabromacetats (X); hier findet Abspaltung von Bromwasserstoff statt (vergl. unten).

Aehnlich wie das Hexabromchinon zeigen auch die beiden Pseudobromide (III und IV) manche Eigenthümlichkeit in ihrem

9) Verläuft die Verseifung des Diacetats aus dem Pentabromchinon (V) in gleicher Weise und kommt diesem wirklich die vorläufig angenommene Formel (vier Bromatome im Kern) zu, so sollte das aus dem Heptabromid dargestellte Phenolderivat VII entstehen. Vorläufige Versuche mit dem Diacetat haben aber nur amorphe Producte ergebell. 
Verhalten. Mit Essigsäureanhydrid reagiren sie in der Kälte und bei Gegeuwart von Schwefelsäure in normaler Weise, es entstehen die entsprechenden Acetylverbindungen:

IS.

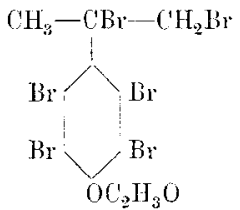

$\mathrm{X}$.

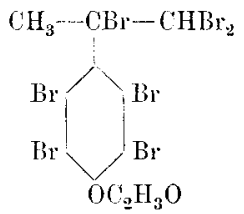

Lässt man dagegen das Essigsäureanhydrid in der Hitze einwirken, so tritt Bromwasserstoff auf und neben den normalen Acetaten (IX und X) erhält man um zwei Bromatome ärmere Acetylderivate, welchen die folgenden Formeln zukommen:
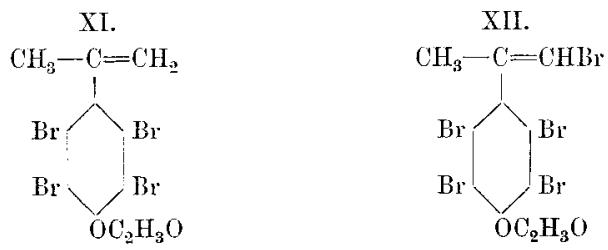

Die Entstehung dieser beiden Verbindungen ist nicht leicht zu erklären. Von einer directen Abspaltung von Brom kann natürlich keine Rede sein, jedenfalls bilden sich zunächst unter Austritt von Bromwasserstoff die Chinone (V und VI) und diese geben dann unter weiterem Bromverlust die Acetylverbindungen XI und XII. Thatsächlich lassen sich, worauf schon oben hingewiesen worden ist, beide Chinone in diese Acetylderivate überführen. Hierbei tritt neben Bromwasserstoff freies Brom auf, was bei den Pseudobromiden nicht der Fall ist; die Ursache dürfte aber wobl eine Nebenreaction sein.

Die Acetylverbindung XII ist identisch mit dem Acetat des Phenols $\mathrm{C}_{9} \mathrm{H}_{5} \mathrm{Br}_{5} \mathrm{O}$ (VII). Das Hexabromchinon (VI) kann also sowohl durch Reduction als auch durch Essigsäureanhydrid in ein bromärmeres ungesättigtes Phenol (VII) übergeführt werden, während es andererseits sich auch ohne Abgabe von Brom in ein ungesättigtes Phenol (VIII) umwandeln kann. 
Ein eigenartig verschiedenes Verhalten zeigen die zuletzt besprochenen vier Acetylderivate beim Verseifen; die aus dem Heptabromid stammenden beiden Derivate (X und XII) lassen sich in alkoholischer Lösung leicht und glatt verseifen. Bei dem Acetylderivat $\mathrm{X}$ findet gleichzeitig Abspaltung von Bromwasserstoff statt, man erhält das Phenol $\mathrm{C}_{9} \mathrm{H}_{4} \mathrm{Br}_{6} \mathrm{O}$ (VIII), während die andere Verbindung das Phenol $\mathrm{C}_{9} \mathrm{H}_{5} \mathrm{Br}_{5} \mathrm{O}$ (VII) liefert.

Aus den beiden vom Hexabromid herstammenden Acetylderivaten IX und XI haben die betreffenden Phenole dagegen noch nicht erhalten werden können. Die Verbindung IX sollte nach den bei dem Heptabromacetat $(\mathrm{X})$ gemachten Beobachtungen das ungesättigte Phenol $\mathrm{C}_{9} \mathrm{H}_{5} \mathrm{Br}_{5} \mathrm{O}$ liefern, statt dessen erhält man beim Verseifen einen in dicken, amorphen Flocken sich abscheidenden Körper. Aebnliche Erscheinungen treten bei der Verseifung der zweiten Acetylverbindung (XI) anf; hier bilden sich auch in Menge amorphe, alkaliunlösliche Producte.

\section{Experimenteller Theil.}

\section{Darstellung des $p$-Diphenoldimethylmethans.}

Man verfährt am besten ${ }^{10}$ ) in der folgenden Weise: Ein Gemisch von $210 \mathrm{~g}$ Phenol, $30 \mathrm{~g}$ Aceton und $21 \mathrm{~g}$ concentrirter Salzsäure $(1,19$ spec. Gew.) wird in einer Stöpselflasche so

$\left.{ }^{10}\right)$ Dianin lässt die Reaction bei $80-90^{\circ}$ vor sich gehen; das Product ist dann weniger rein und die Ausbeute geringer.

Auch die drei Kresole condensiren sich sehr leicht mit Aceton; o-Kresol liefert dabei das dem Phenolderivat entsprechende Dikresoldimethylmethan (Unverzagt, Dissert. Marburg 1904), wihrend $p$ - und $m$-Kresol in ganz anderer Weise reagiren. Die Condensation verkiuft hier nach der Gleichnng:

$$
\mathrm{C}_{6} \mathrm{H}_{4}(\mathrm{OH}) \mathrm{CH}_{3}+\mathrm{C}_{3} \mathrm{H}_{6} \mathrm{O}=\mathrm{C}_{10} \mathrm{H}_{12} \mathrm{O}+\mathrm{H}_{2} \mathrm{O} \text {. }
$$

Den entstehenden Verbindungen kommt aber die verdoppelte Formel zu, sie sind alkaliunlöslich und sehr beständig (Ga a bel, Dissert. Marburg 1903). 
lange bei $30-40^{\circ}$ stehen gelassen, bis die ganze Masse krystallinisch erstarrt ist, was nach zwei bis drei Tagen der Fall sein wird. Man rührt dann mit $300 \mathrm{ccm} 35-40$ proc. Essigsäure gut durch, saugt $a b$ und wäscht mit verdünnter Essigsäure und schliesslich mit Wasser nach. Die Ausbeute beträgt $120 \mathrm{~g}$; das Product besteht aus kleinen, weissen Kryställchen, enthält aber noch etwas Phenol, von dem es durch Trocknen bei $100^{\circ}$ befreit werden kann. Zur weiteren Reinigung, die aber für die hier in Betracht kommenden Versuche unnöthig ist, krystallisirt man aus heisser verdünnter Essigsäure um. Die Verbindung bildet farblose Nadeln oder Prismen vom Schmelzpunkt $152-153^{\circ}$, sie ist leicht löslich in Allohol, Aether, Eisessig und Benzol. Die vom Rohproducte abgesaugte Lauge enthält neben dem überschüssigen Phenol nur noch wenig Diphenoldimethylmethan, ihre Verarbeitung lohnt nicht.

Bei Darstellungen in grösserem Maassstabe wird man mit der berechneten Menge von Phenol arbeiten können; das Product fällt dann weniger rein aus. Die Ausbeute ist aber auch gut.

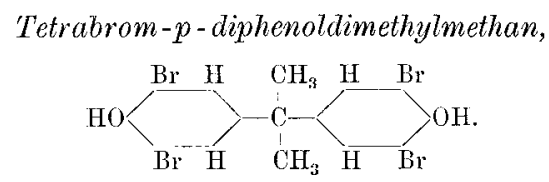

Das Diphenoldimethylmethan wird in fünf Theilen Eisessig gelöst und die Lösung kalt mit drei Theilen Brom versetzt. Beim Stehen scheidet sich das Tetrabromid in dicken Krystallen ab, die nach ein oder zwei Tagen abgesangt und mit verdünnter Essigsäure ausgewaschen werden. Für die meisten Verarbeitungen ist das so erhaltene Product ausreichend rein. Die Lauge lässt man etwas abziehen und fällt dann vorsichtig mit wenig Wasser aus. Zur Reinigung wird aus Essigsäure umkrystallisirt. Die Ausbente beträgt bis $90 \mathrm{pC}$. der berechneten.

Das Tetrabromderivat krystallisirt aus heisser Essigsäure in schönen, glänzenden Spiessen und Prismen vom Schmelzpunkt $162-163^{\circ}$. In Aether, Chloroform, Aceton, Benzol und 
Alkohol ist es leicht löslich, weniger leicht in Essigsäure; Benzin löst es sehr schwer; von Alkali wird es leicht und ohne Veränderung gelöst, von Soda beim Erwärmen ebenfalls leicht.

I. $0,1576 \mathrm{~g}$ gaben $0,1909 \mathrm{CO}_{2}$ und $0,0361 \mathrm{H}_{2} \mathrm{O}$.

II. $0,1700 \mathrm{~g} \quad, \quad 0,2364 \mathrm{AgBr}$.

\begin{tabular}{lcccc} 
& Berechnet für & \multicolumn{2}{c}{ Gefunden } \\
& $\mathrm{C}_{15} \mathrm{H}_{12} \mathrm{Br}_{4} \mathrm{O}_{2}$ & I. & II. \\
$\mathrm{C}$ & 33,09 & 33,04 & - \\
$\mathrm{H}$ & 2,22 & 2,56 & - \\
$\mathrm{Br}$ & 58,88 & - & 59,18
\end{tabular}

Acetylverbindung. Mit Essigsäureanhydrid und Natriumacetat dargestellt und aus Eisessig umkrystallisirt. Derbe, weisse Krystallnadeln, bei $169-170^{\circ}$ schmelzend, in Aceton, Benzol und heissem Eisessig leicht löslich, weniger in Aether und Alkohol.

I. $0,1850 \mathrm{~g}$ gaben $0,2469 \mathrm{CO}_{2}$ und $0,0436 \mathrm{H}_{2} \mathrm{O}$.

II. $0,1308 \mathrm{~g} \quad " \quad 0,1578 \mathrm{AgBr}$.

\begin{tabular}{lccc} 
& Berechnet für & \multicolumn{2}{c}{ Gefunden } \\
$\mathrm{C}$ & $\mathrm{C}_{19} \mathrm{H}_{16} \mathrm{Br}_{4} \mathrm{O}_{4}$ & I. & II. \\
$\mathrm{C}$ & 36,31 & 36,40 & - \\
$\mathrm{II}$ & 2,57 & 2,64 & - \\
$\mathrm{Br}$ & 50,93 & - & 51,34
\end{tabular}

Dibromdinitro- $p$-diphenoldimethylmethan,<smiles></smiles>

Das Tetrabromid wird in Eisessig gelöst und die Lösung in der Kälte nach und nach mit überschüssigem festem Natriumnitrit versetzt. Man lässt dann einige Zeit stehen, fällt die Nitroverbindung vorsichtig mit Wasser aus und krystallisirt aus verdünntem Eisessig um.

Das Dibromdinitroderivat bildet gelbe Nadeln vom Schmelzpunkt $176^{\circ}$; in Alkohol, Aether, Eisessig und Benzol ist es leicht, in Benzin sehr schwer löslich. Das Ammonsalz und die Alkalisalze sind roth gefürbt und in Wasser schwer löslich. 
88 Zincke, Einuirkung von Brom und von Chlor auf Phenole.

I. $0,1620 \mathrm{~g}$ gaben $8,5 \mathrm{ccm}$ Stickgas bei $16^{\circ}$ und $744 \mathrm{~mm}$ Druck.

II. $0,1187 \mathrm{~g}, \quad 0,0943 \mathrm{AgBr}$.

\begin{tabular}{cccc} 
& Berechnet fïr & \multicolumn{2}{c}{ Gefunden } \\
& $\mathrm{C}_{15} \mathrm{H}_{12} \mathrm{Br}_{9} \mathrm{~N}_{2} \mathrm{O}_{6}$ & I. & II \\
$\mathrm{N}$ & 5,90 & 6,02 & - \\
$\mathrm{Br}$ & 33,59 & -- & 33,81
\end{tabular}

Acetylverbindung. Mit Anhydrid und Natriumacetat dargestellt und aus verdünnter Essigsäure umkrystallisirt. Schwefelgelbe, dicke Nädelchen vom Schmelzp. 196-197\%.

$0,1300 \mathrm{~g}$ gaben $0,0863 \mathrm{AgBr}$.

$\begin{array}{cc}\text { Berechnet fiirr } & \text { Gefunden } \\ \mathrm{C}_{19} \mathrm{H}_{19} \mathrm{Br}_{2} \mathrm{~N}_{2} \mathrm{O}_{8} & \\ 28,55 & 28,2 \check{5}\end{array}$

\section{Einwirkung von Brom anl' Tetrabromdiphenoldimethymethan.}

Heptabrompseudobromid und Umwandlungsproducte.

Die Einwirkung von Brom auf das Tetrabromderivat ohne Lösungsmittel führt sofort Spaltung des Moleküls herbei; man erhält Tribromphenol und je nach den Bedingungen ein Pseudoheptabromid oder ein Pseudohexabromid des Isopropylphenols. Ersteres ist eingehend untersucht worden, seine Coustitution erscheint uns nicht zweifelhaft (vergl. die Einleitung).

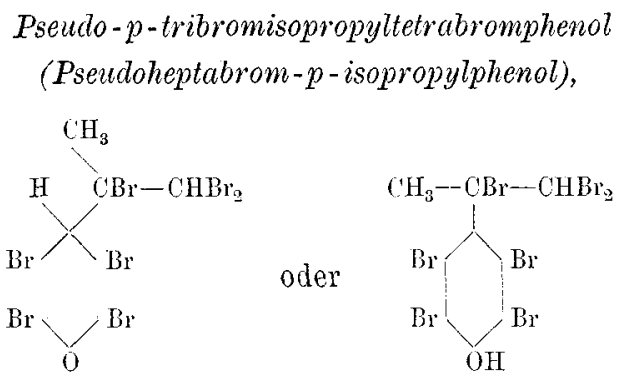

Diese Verbindung entsteht, wenn das Tetrabromdiphenoldimethylmethan mit überschüssigem Brom behandelt wird; man 
stellt sie am besten im geschlossenen Rohre bei $100^{\circ}$ dar. Ein Theil Tetrabromid wird mit zwei Theilen Brom übergossen, die Mischung 10--12 Stunden bei gewöhnlicher Temperatur stehen gelassen, die Röhre dann zugeschmolzen und drei Stunden auf $100^{\circ}$ erhitzt. Der Inhalt der Röhren wird mit etwas warmem Benzol herausgespült, Benzol und überschüssiges Brom verdunsten gelassen und der Rückstand nach dem Trocknen im Vacuum wiederholt mit heissem Benzin ausgezogen, um Tribromphenol zu entfernen. Hierbei löst sich auch etwas Heptabromid, man kann die Benzinlauge auf das Hexabromchinon verarbeiten ${ }^{1}$ ). Der vom Benzin nicht gelöste Theil wird aus einem Gemisch von Benzin mit Benzol umkrystallisirt. Die Ausbeute beträgt $79-80$ pC. der berechneten.

Das Heptabrompseudobromid krystallisirt in schönen, glänzenden, dicken Nadeln oder Prismen von monoklinem Habitus; es schmilzt unter Röthung und Zersetzung bei $182-183^{\circ}$. In Aceton ist es leicht löslich, auch heisses Benzol und heisser Eisessig lösen es leicht, in Aether ist es schwerer löslich, in Benzin nur in sehr geringer Menge.

Zur Analyse wurde im Vacuum bei $50-60^{\circ}$ getrocknet.

I. $0,2303 \mathrm{~g}$ gaben $0,1348 \mathrm{CO}_{2}$ und $0,214 \mathrm{H}_{2} \mathrm{O}$.

II. $0,1400 \mathrm{~g} \quad, \quad 0,2670 \mathrm{AgBr}$.

III. $0,1231 \mathrm{~g} \quad, \quad 0,2348 \mathrm{AgBr}$.

11) Beim Abdunsten hinterlässt die Lösung eine krystallinische Nasse, welche mit Natronlange ein grünes Natriumsalz giebt, während Tribromphenol in Lösung geht, das grüne Salz dürfte wohl eine Chinhydronverbindung sein, entstanden durch Finwirkung yon Tribromphenol auf das Hexabromchinon, welches aus dem gleichzeitig vorhandenen Heptabromid entsteht. Thatsächlich erhält man ein griines Natriumsalz, wenn Tribromphenol mit Hexabromchinon und etwas Natronlange zusammengerieben wird. Das im obigen Abdampfungsrïckstande enthaltene Tribromphenol kann durch Auszieben mit Soda und Umkrystallisiren aus Benzin rein erhalten werden (Schmelzp. 91-92 ; gefunden $21,60 \mathrm{C}$ und $0,99 \mathrm{H}$, berechnet $21,76 \mathrm{C}$ and $0,91 \mathrm{H}$ ). 


\begin{tabular}{ccccc} 
& Berechnet für & \multicolumn{3}{c}{ Gefunden } \\
\cline { 3 - 5 } & $\mathrm{C}_{9} \mathrm{H}_{5} \mathrm{Br}_{7} \mathrm{O}$ & I. & II. & III. \\
$\mathrm{C}$ & 15,68 & 15,96 & - & - \\
$\mathrm{H}$ & 0,73 & 1,04 & - & - \\
$\mathrm{Br}$ & 81,27 & - & 81,15 & 81,17
\end{tabular}

III aus dem Hexabromchinon durch Addition von Bromwasserstoff dargestellt (vergl. unten).

In seinem allgemeinen Verhalten gleicht das Heptabromid den früher beschriebenen Pseudobromiden. In Acetonlösung tritt auf Zusatz von Wasser sofort Bildung von Hexabromchinon ein; auch beim Behandeln mit Eisessig und mit Alkoholen dürfte zunächst Chinonbildung stattfinden, doch sind diese Reactionen nicht weiter verfolgt. Mit Essigsäureanhydrid und etwas Schwefelsäure in der Kälte behandelt entsteht die normale Heptabromacetylverbindung. Lässt man das Anhydrid aber in der Hitze einwirken, so findet Abspaltung von Bromwasserstoff statt und man erhält neben der normalen Verbindung ein Pentabromacetat. (Vergl. unten.)

Alkali wirkt auf das Heptabromid in der Kälte kaum ein, beim Erwärmen findet langsam Lösung statt, wobei wohl zunächst Chinon entsteht, das dann weiter reagirt. Beim Erhitzen mit Salpetersäure $(1,4)$ tritt Abspaltung von Brom und Oxydation ein, es bildet sich aber kein Tetrabrombenzochinon, sondern ein weisser, krystallinischer Körper, welcher mit Soda tief gelb wird und allmählich in Lösung geht.

Acetylderivat des Heptabrom-p-isopropylphenols,<smiles></smiles>

Die Darstellung dieser Verbindung gelingt glatt nur in der Kälte ${ }^{12}$ ) bei Gegenwart von etwas concentrirter Schwefel-

32) In der Wärme entstelt gleichzeitig eine Pentabromverbindung (vergl. unten). 
säure; das Pseudobromid reagirt dann wie ein Phenol, scheinbar geht es, ohne dass Chinon als Zwischenproduct sich bildet, in das Acetylderivat über.

Man übergiesst das feingepulverte Heptabromid mit Essigsäureanhydrid und setzt etwas Schwefelsäure zu; auf $2 \mathrm{~g}$ Heptabromid $5 \mathrm{ccm}$ Anhydrid und fünf Tropfen Säure. Beim Schütteln geht das Pseudobromid langsam in Lösung und bei längerem Stehen scheidet sich ein Theil der Acetylverbindung in kleinen Kryställchen aus. Man giesst ab, wäscht mit etwas Alkohol nach und krystallisirt aus heissem Alkohol um. Die abgegossene Flüssigkeit wird mit Wasser gefällt, das ausgeschiedene Product mit Alkohol angerieben, ausgepresst und dann ebenfalls umkrystallisirt.

Die Acetylverbindung bildet farblose, dicke Nadeln vom Schmelzp. $166^{\circ}$; sie ist in Aceton, Eisessig, Benzol ziemlich löslich, namentlich in der Wärme, schwieriger löst sie sich in Alkohol; in Soda und verdünnter Natronlauge ist sie unlöslich.

$0,1525 \mathrm{~g}$ gaben $0,2745 \mathrm{AgBr}$.

$$
\begin{array}{ccc} 
& \text { Berechnet für } & \text { Gefunden } \\
& \mathrm{C}_{\mathbf{1 1}} \mathrm{H}_{7} \mathrm{Br}_{7} \mathrm{O}_{2} & \\
\mathrm{Br} & 76,59 & 76,60
\end{array}
$$

Mit Alkali in alkoholischer Lösung lässt sich diese Acetylverbindung unter Abgabe von Bromwasserstoff glatt verseifen; es entsteht ein Hexabromisopropenylphenol, welches isomer mit dem Hexabromehinon ist und auch aus diesem erhalten werden kann. Es ist unten näher beschrieben.

$p$-Dibromisopropylidentetrabromchinon

(Hexabromisopropylidenchinon),

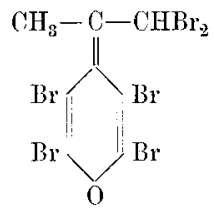

Die Darstellung dieses Chinons ist sebr leicht. Das Heptabromid wird in fünf Theilen Aceton gelöst und nach und nach 
drei Theile Wasser zugesetzt. Es findet sofort Abspaltung von Bromwasserstoff statt, die Lösung färbt sich intensiy gelb und scheidet, ehe alles Wasser zugesetzt ist, Chinon in schön gelben Krystallnädelchen ab, deren Menge bei der weiteren Verdünnung zunimmt. Man saugt sofort ab und trocknet über Schwefelsäure. Die Ausbeute beträgt $80-85$ pC. der berechneten.

Auch die Benzinlaugen von der Darstellung des Heptabromids lassen sich noch auf Chinon verarbeiten; man verdünnt mit dem gleichen Volumen Aether, schüttelt mit einer zehnprocentigen Sodalösung gut durch und lässt die Aether-Benzinlösung verdunsten. Das so erhaltene Chinon wird nach dem Waschen mit etwas Aether durch Umkrystallisiren aus BenzolBenzin gereinigt.

Das Hexabromisopropylidenchinon ist sehr beständig, es lässt sich sogar aus heissem Eisessig umkrystallisiren. Besser eignet sich zu diesem Zwecke aber ein Gemisch von Benzol und Benzin, aus welchem das Chinon in schönen, glänzenden, dunkelgelben Nadeln oder auch in gut ausgebildeten dicken Prismen vom Schmelzp. $185^{\circ}$ sich abscheidet. In warmem Aceton, Chloroform und Benzol ist es leicht löslich, schwieriger löst es sich in Aether, noch weniger in Benzin.

I. $0,2151 \mathrm{~g}$ gaben $0,1416 \mathrm{CO}_{2}$ und $0,0157 \mathrm{H}_{2} \mathrm{O}$.

II. $0,1123 \mathrm{~g} \quad, \quad 0,2078 \mathrm{AgBr}$.

III. $0,1441 \mathrm{~g} \quad, \quad 0,2677 \mathrm{AgBr}$.

\begin{tabular}{ccccc} 
& Berechnet für & \multicolumn{4}{c}{ Gefunden } \\
\cline { 3 - 6 } & $\mathrm{C}_{9} \mathrm{H}_{4} \mathrm{Br}_{8} \mathrm{O}$ & I. & II. & III. \\
$\mathrm{C}$ & 17,76 & $17,9 \tilde{5}$ & -- & - \\
$\mathrm{H}$ & 0,66 & 0,82 & - & - \\
$\mathrm{Br}$ & 78,94 & - & 78,74 & 79,06
\end{tabular}

Das Hexabromchinon zeigt verschiedene eigenartige Reactionen. Von Essigsäureanhydrid wird es bei Gegenwart von Schwefelsäure in der Kälte in das Diacetat des zugehörigen Phenolalkohols übergeführt; beim Erhitzen mit dem Anhydrid bildet sich dagegen unter Freiwerden von Brom und Bromwasserstoff das Acetylderivat eines Pentabromisopropenylphenols. 
Dieses Phenol kann auch direct aus dem Chinon durch Reduction gewonnen werden; andererseits geht das Chinon leicht in das isomere Hexabromisopropenylphenol über, so namentlich mit Alkali bei Gegenwart von Aceton oder Alkohol. Von Sodalösung wird das Chinon kaum angegriffen, wässriges Alkali löst es langsam. Eine Addition von Wasser oder von Alkohol ist bis jetzt nicht gelungen. Bromwasserstoff führt es wieder in das Psendobromid über. Die in Betracht kommenden Umwandlungsproducte sind unten beschrieben.

1-Dibrom - 2-acetoxy-p-isopropyltetrabromphenolacetat,<smiles>CCOC(Br)(Br)C(Br)C(Br)C(Br)Br</smiles>

Man übergiesst das Hexabromchinon mit zwei bis drei Theilen Essigsäureanhydrid und setzt etwas concentrirte Schwefelsäure zu. Das Chinon löst sich allmählich auf und aus der farblosen Lösung scheidet sich beim Stehen das Diacetat in kleinen Kryställchen aus. Man saugt ab, wäscht mit etwas Alkohol nach und krystallisirt aus heissem verdünnten Alkohol um.

Das Diacetat bildet schöne glänzende Prismen vom Schmelzpunkt $144-145^{\circ}$. In Aceton ist es leicht löslich; auch Benzol und Alkohol lösen es in der Wärme leicht, weniger Aether und noch weniger Benzin.

I. $0,3141 \mathrm{~g}$ gaben $0,2535 \mathrm{CO}_{2}$ und $0,0409 \mathrm{H}_{2} \mathrm{O}$.

II. $0,1502 \mathrm{~g} \quad, \quad 0,2391 \mathrm{AgBr}$.

III. $0,1070 \mathrm{~g} \quad, \quad 0,1696 \mathrm{AgBr}$.

\begin{tabular}{ccccc} 
& Berechnet fïr & \multicolumn{3}{c}{ Gefunden } \\
& $\mathrm{C}_{13} \mathrm{H}_{10} \mathrm{Br}_{6} \mathrm{O}_{4}$ & I. & II. & III. \\
$\mathrm{C}$ & 21,98 & 22,01 & - & - \\
$\mathrm{H}$ & 1,42 & 1,46 & - & - \\
$\mathrm{Br}$ & 67,59 & - & 67,74 & 67,45
\end{tabular}


94 Zincke, Einwirkung von Brom und von Chlor auf Phenole.

Bei der Verseifung in alkoholischer Lösung liefert das Diacetat nicht den zugehörigen Phenolalkohol, man erhält vielmehr in guter Ausbeute Hexabromisopropenylphenol. (Siehe die folgende Verbindung.)

Der Phenolalkohol,<smiles>CC(O)C(Br)C(Br)Br</smiles>

ist augenscheinlich sehr geneigt, Wasser abzuspalten und das dürfte auch der Grund sein, weshalb er so schwer zu erhaiten ist. Neben anderen Producten haben wir ihn in geringer Menge bei der Einwirkung von concentrirter Schwefelsäure auf eine heisse Lösung des Chinons in Eisessig erhalten. Durch vorsichtige fractionirte Fällung mit Wasser erbält man zuletzt kleine, weisse, alkalilösliche Krystallnadeln vom Schmelzp. $114^{0}$ bis $117^{\circ}$. Der Bromgehalt betrug 76,28 pC., während sich für den Phenolalkohol - Addition von Wasser an das Chinon - 76,66 pC. berechnen. Mit Essigsäureanhydrid und Schwefelsäure gehen die Nadeln in das oben beschriebene Diacetat über. Analyse I ist mit so dargestelltem Material ausgeführt.

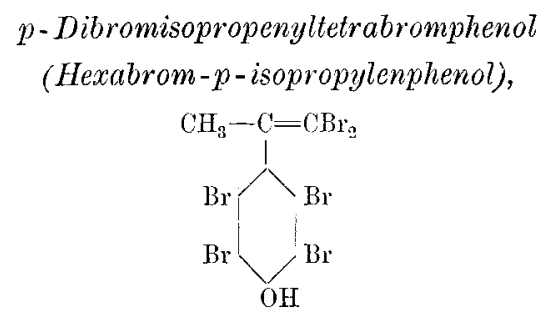

Diese mit dem Hexabromchinon isomere Verbindung lässt sich am leichtesten durch Verseifen des oben beschriebenen

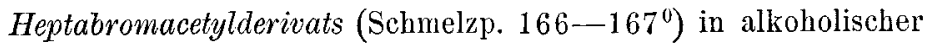
Lösung darstellen. Die Verseifung tritt in der Wärme rasch ein, man verdünnt dann mit Wasser, fällt mit Salzsäure aus und krystallisirt aus Eisessig um. 
Aus dem isomeren Chinon entsteht das Phenolderivat bei Reactionen, die eigentlich zu dem sogenannten Wasserproduct, dem Phenolalkohol (vergl, oben) hätten führen sollen, so z. B. bei der Verseifung des oben beschriebenen aus dem Chinon dargestellten Diacetats, bei der Einwirkung von Alkali in Aceton- oder Methylalkohollösung auf das Chinon, ebenso wenn an Stelle von Alkali eine alkalische Zinnchlorürlösung angewendet wird. Auch beim Kochen mit Aceton oder Alkohol unter Zusatz von etwas gelbem Quecksilberoxyd geht das Chinon in das isomere Phenolderivat über. Scheinbar handelt es sich bei diesen Reactionen nur um eine Umlagerung des Chinons. (Vergl. hierüber die Einleitung.)

Am glattesten geht die directe Umwandlung des Chinons in das Phenolderivat mit alkalischer Zinnlösung vor sich. Man löst das Chinon in kaltem Aceton und setzt Zinnlösung $\mathrm{zu}$, bis vollständige Entfärbung eingetreten ist. Dann wird mit Salzsäure angesäuert, das Aceton theilweise verdunsten gelassen, mit Wasser verdünnt, abfiltrirt und aus Eisessig umkrystallisirt.

Das Hexabromisopropylenphenol krystallisirt in langen, weissen Nadeln rom Schmelzp. 134-135 ; in Aceton und in dether ist es leicht löslich, nicht ganz so leicht in Alkobol, Eisessig, Benzol, in Benzin schwer löslich. Mit Natronlauge entsteht ein in Alkali schwer lösliches weisses Natriumsalz.

ฯ. $0,1935 \mathrm{~g}$ gaben $0,1267 \mathrm{CO}_{2}$ und $0,0159 \mathrm{H}_{2} \mathrm{O}$.

II. $0,2042 \mathrm{~g} \quad, \quad 0,1333 \quad \mathrm{CO}_{\mathrm{z}}, 0,0166 \mathrm{H}_{2} \mathrm{O}$.

III. $0,1575 \mathrm{~g} \quad, \quad 0,2918 \mathrm{AgBr}$.

IV. $0,1712 \mathrm{~g} \quad, \quad 0,3181 \mathrm{AgBr}$.

V. $0,1582 \mathrm{~g} \quad, \quad 0,2932 \mathrm{AgBr}$.

\begin{tabular}{ccccccc} 
& Berechuet fiir & \multicolumn{7}{c}{ Gotinden } \\
\cline { 3 - 7 } & $\mathrm{C}_{9} \mathrm{H}_{4} \mathrm{Br}_{6} \mathrm{O}$ & I. & II. & III. & IV. & V. \\
$\mathrm{C}$ & 17,76 & 17,86 & 17,80 & - & - & - \\
$\mathrm{H}$ & 0,66 & 0,92 & 0,91 & - & - & - \\
$\mathrm{Br}$ & 78,94 & - & - & 78,84 & 79,00 & 78,87
\end{tabular}

I mit Quecksilberoxyd in Acetonlösung dargestellt, II und III mit alkalischer Zimnlösung, IV durch Verseifen des Heptabromacetats, $V$ aus dem Chinondiacetat. 
In Eisessiglösung mit Bromwasserstoff behandelt, geht das Propenylphenol wieder in das Pseudoheptabromid über; auch Brom wird addirt unter Bildung eines Pseudobromids, das aber nicht untersucht worden ist. Mit Salpetersäure (1,4 spec. Gew.) entstehen gelbe, harzige Producte, die durch Eisessig weiss und krystallinisch werden und sich mit Soda intensiv gelb färben.

Acetylverbindung. Mit Essigsäureanhydrid und Schwefelsäure sowie durch Erwärmen mit Acetylbromid dargestellt und durch Umkrystallisiren aus Benzin gereinigt. Schöne, glänzende, monokline Prismen vom Schmelzp. $114-115^{\circ}$; in Aether, Alkohol, Eisessig ziemlich leicht löslich.

I. $0,2061 \mathrm{~g}$ gaben $0,1539 \mathrm{CO}_{2}$ und $0,0209 \mathrm{H}_{2} \mathrm{O}$

II. $0,1453 \mathrm{~g} \quad, \quad 0,2508 \mathrm{AgBr}$.

III. $0,1042 \mathrm{~g} \quad, \quad 0,1805 \mathrm{AgBr}$.

\begin{tabular}{lccccc} 
& Berechnet füir & \multicolumn{3}{c}{ Gefunden } \\
\cline { 3 - 6 } & $\mathrm{C}_{11} \mathrm{H}_{6} \mathrm{Br}_{6} \mathrm{O}_{2}$ & I. & II. & III. \\
$\mathrm{H}$ & 20,32 & 20,37 & - & - \\
$\mathrm{Br}$ & 0,93 & 1,13 & - & -
\end{tabular}

I und II mit Essigsäureanhydrid, III mit Acetylbromid dargestellt.

p-Bromisopropenyltetrabromphenol

(Pentabrom-p-isopropylenphenol),<smiles>CC(=CBr)C(Br)C(Br)Br</smiles>

Entsteht aus dem Hexabromisopropylidenchinon, wenn dieses in Acetonlösung etwa zwei Tage stehen bleibt, die Lösung ist dann farblos geworden und giebt mit Wasser eine ölige Ausscheidung, welche langsam fest wird. Rascher erfolgt die Umwandlung beim Kochen der Acetonlösung, in etwa einer halben Stunde ist die Gelbfärbung verschwunden. Das Aceton wirkt hier als Reductionsmittel, was nicht der Fall ist, wenn Quecksilberoxyd zugesetzt wird (vergl. die vorhergehende Verbindung). 
Auf diese Weise lässt sich die Verbindung aber nur sehr schwer rein erhalten, man stellt sic besser durch Einwirkung von Zinnchlorür auf das Hexabromchinon dar. Ein Theil Chinon wird mit fünf Theilen Eisessig erwärmt und so lange Zinnchlorürlösung ${ }^{13}$ ) zugefügt, bis vollständige Entfärbung eingetreten ist. Beim Erkalten scheidet sich das entstandene Product in Nadeln aus, durch Umkrystallisiren aus Eisessig wird es gereinigt.

In Form der Acetylverbindung erhält man das Propenylderivat, wenn das Pseudoheptabromid oder das Hexabromchinon mit Essigsäureanhydrid gekocht wird; das Acetylderivat lässt sich leicht verseifen.

Das Pentabromisopropylenphenol krystallisirt in langen, harten, weissen Nadeln vom Schmelzp. 88-890. In Alkohol, Eisessig, Benzin ist es in der Hitze leicht löslich, viel weniger löst es sich in der Kälte. Mit Natronlauge entsteht ein weisses, gut krystallisirendes Natriumsalz.

I. 0,2594 graben $0,1952 \mathrm{CO}_{2}$ und $0,0239 \mathrm{H}_{2} \mathrm{O}$.

II. $0,1195 \mathrm{~g} \quad " \quad 0,2122 \mathrm{\Lambda gBr}$.

III. $0,1187 \mathrm{~g} \quad, \quad 0,2124 \mathrm{AgBr}$.

\begin{tabular}{lcccc} 
& Berechnet fiur & \multicolumn{3}{c}{ Gefunden } \\
\cline { 3 - 6 } & $\mathrm{C}_{9} \mathrm{H}_{5} \mathrm{Br}_{5} \mathrm{O}$ & I. & II. & III. \\
$\mathrm{C}$ & 20,42 & 20,52 & - & - \\
$\mathrm{H}$ & 0,95 & 1,03 & - & - \\
$\mathrm{Br}$ & 75,60 & - & 75,57 & 76,18
\end{tabular}

I und II mit Zinnehloriir dargestellt, III durch Erhitzen mit Aceton.

In Eisessiglösung mit Brom behandelt, geht das Propenylderivat in das Heptabrompseudobromid über, mit Bromwasserstoff entsteht neben einem Pseudobromid eine alkalilösliche Verbindung. (Vergl. die Einleitung.)

Sehr eigenartig ist das Verhalten gegen Salpetersäure (1,4 spec. Gew.) beim Erwärmen, ohne dass Oxydation stattfindet, geht eine Umwandlung in schöne gelbe Kryställchen

13) Durch Liosen von Zim in concentrirter Salzsiure dargestellt. $1 \mathrm{cem}=0,25 \mathrm{Sn}$. 
vor sich. Beim Erhitzen spaltet dieses Product rothe Stickoxyde ab, in kaltem Alkali löst es sich langsam und unter Zersetzung auf.

Acetylverbindung. Mit Hülfe von Essigsäureanhydrid und etwas concentrirter Schwefelsäure leicht darstellbar. Bildet sich auch beim Kochen des Heptabrompseudobromids und des Hexabromchinons mit Essigsäureanhydrid (vergl, unten). Sie krystallisirt ausgezeichnet und kann aus Benzin in grossen, glänzenden, monoklinen Prismen erhalten werden. Benzin, Eisessig und Alkohol lösen sie in der Hitze leicht, viel weniger in der Kälte. Der Schmelzpunkt liegt bei $126-127^{\circ}$.

I. $0,2189 \mathrm{~g}$ gaben $0,1867 \mathrm{CO}_{2}$ und $0,0253 \mathrm{H}_{2} \mathrm{O}$.

II. $0,1342 \mathrm{~g} \quad$, $\quad 0,2212 \mathrm{AgBr}$.

\begin{tabular}{cccc} 
& Berechnet für & \multicolumn{2}{c}{ Gefunden } \\
& $\mathrm{C}_{11} \mathrm{H}_{7} \mathrm{Br}_{5} \mathrm{O}_{2}$ & J. & II. \\
$\mathrm{C}$ & 23,12 & 23,26 & - \\
$\mathrm{H}$ & 1,24 & 1,29 & - \\
$\mathrm{Br}$ & 70,04 & - & 70,14
\end{tabular}

Die Analysen des aus Heptabromid und aus Hexabromchinon dargestellten Productes finden sich im folgenden Abschnitte.

Einwirkung von Essigsäureanhydrid in der Hitze auf Heptabrompseudobromid und auf Hexabromchinon.

Bildung von $p$-Bromisopropenyltetrabromphenolacetat,<smiles>CC(=O)OC1C(Br)C(Br)C(Br)C1Br</smiles>

Die sich hier abspielenden Reactionen sind bereits in der Einleitung besprochen worden, so dass die Angabe der experimentellen Daten genügt.

Man kocht das Heptabrompseudobromid mit der 2- bis 2,5 fachen Menge Essigsäureanhydrid 1/4 Stunde lang; es entweicht Bromwasserstoff und die Lösung nimmt eine gelbe Farbe an, wird später aber wieder heller. Beim Stehen der erkalteten Lösung scheidet sich ein Theil des entstandenen Pentabrom- 
acetats aus. Man giesst $a b$ und lässt das Essigsäureanhydrid abdunsten; es hinterbleibt ein krystallinischer Rückstand, welcher beim Umkrystallisiren ans Eisessig zunächst das bei $166-167^{\circ}$ schmelzende Heptabromacetat giebt. Aus der Lauge lassen sich aber durch Abdunsten, Auslesen der Krystalle und Umkrystallisiren aus Benzin noch weitere Mengen des Pentabromacetats gewinnen. Aus $10 \mathrm{~g}$ Heptabromid wurden auf diese Weise in Ganzen $3 \mathrm{~g}$ erhalten.

In gleicher Weise verfährt man mit dem Hexabromchinon, die Lösung färbt sich hier bräunlich, hellt später aber. wieder etwas auf. Entweichen von Bromwasserstoff und Brom ist deutlich wahrnehmbar. Beim Erkalten scheidet sich ein Theil des Pentabromacetats aus; die Lauge hinterlässt beim Abdunsten ein dickes Harz, aus dem aber durch Lösen in Aether, fractiovirtes Fällen mit Benzin und Umkrystallisiren der krystallinischen Antheile noch reines Product gewonnen werden kann. Die Ausbente kommt der aus dem Heptabromid erhaltenen ungefähr gleich.

Das auf diese Weise erhaltene Acetat stimmt in Zusammensetzung und Eigenschaften mit dem vorhin beschriebenen Pentabromacetat völlig überein.

I. $0,1883 \mathrm{~g}$ gaben $0,1568 \mathrm{CO}_{\mathrm{g}}$ und $0,0230 \mathrm{H}_{2} \mathrm{O}$.

II. $0,1110 \mathrm{~g} \quad, \quad 0,1825 \mathrm{AgBr}$.

III. $0,1772 \mathrm{~g} \quad, \quad 0,2912 \mathrm{AgBr}$.

\begin{tabular}{ccccc} 
& Berechnet fuir & \multicolumn{3}{c}{ Gefunden } \\
\cline { 3 - 6 } & $\mathrm{C}_{11} \mathrm{H}_{7} \mathrm{Br}_{5} \mathrm{O}_{2}$ & I. & II. & III. \\
$\mathrm{C}$ & 23,12 & 22,72 & - & - \\
$\mathrm{H}$ & 1,24 & 1,37 & - & - \\
$\mathrm{Br}$ & 70,04 & - & 69,98 & 69,94
\end{tabular}

I und II aus Heptabromid, III aus Chinon dargestellt.

Wird das Acetat in alkoholischer Lösung mit etwas Alkali erwärmt, so tritt rasch Verseifung ein; das entstehende Product stimmt vollständig mit dem oben beschriebenen Bromisopropenyltetrabromphenol überein. 